\title{
Physician Awareness in Diabetes Management During Ramadan 2015-A Focus Group Discussion
}

\author{
Fatima Younis Al Slail,, ${ }^{1}$ Haroon UR Rashid, ${ }^{2}$ Sahar Mohamed Fadl, ${ }^{1}$ and Omer Osman Kheir,
}

1. National Diabetes and Prevention Program, Ministry of Health, Riyadh, Saudi Arabia; 2. World Health Organization, Libya

$\mathrm{B}$ ackground: Diabetic Muslims who wish to fast are recommended to undergo medical counseling prior to fasting to prevent complications. This study assesses knowledge, attitudes, and practices, and identifies issues related to building capacity for physicians treating diabetic patients. Methods: We conducted a qualitative study based on focus group discussions with primary healthcare center (PHCC) doctors who manage diabetic patients in the Riyadh region. Results: There is a lack of knowledge of the classification system for risk assessment of diabetic patients who fast during Ramadan. All the responses showed that there were misconceptions regarding nitroglycerin tablets placed under the tongue to nullify fasting. Other issues addressed by respondents include the following: how to adjust the dose and subsequently convince the patient to follow a new regimen, loss of patient follow-up due to referral to the hospital, the refusal of some laboratories to perform examinations for patients referred from other PHCCs, and lack of patient medication compliance. Conclusion: The study reported a lack of knowledge among respondents regarding therapeutic and lifestyle management of diabetic patients during Ramadan. Other issues addressed by participants include lack of healthcare services at primary healthcare center facilities and services not working effectively. To empower physicians and improve knowledge, attitude, and practices for managing diabetic patients during Ramadan, experience, continuous training, as well as fully equipped healthcare centers (including both laboratory and pharmaceutical medical supplies) play a crucial role.

\section{Keywords}

Diabetes, Ramadan, management, physician, focus group, primary healthcare center, type 2 diabetes, fasting

Disclosure: Fatima Younis Al Slail, Haroon UR Rashid, Sahar Mohamed Fadl, and Omer Osman Kheir have nothing to declare in relation to this article. This work was funded by the National Diabetes Prevention and Control Program, Ministry of Health, Saudi Arabia. There were no publication fees associated with the publication of this article.

Compliance with Ethics: All procedures were followed in accordance with the responsible committee on human experimentation and with the Helsinki Declaration of 1975 and subsequent revisions.

Authorship: All named authors meet the International Committee of Medical Journal Editors (ICMJE) criteria for authorship of this manuscript, take responsibility for the integrity of the work as a whole, and have given final approval to the version to be published.

open Access: This article is published under the Creative Commons Attribution Noncommercial License, which permits any noncommercial use, distribution, adaptation, and reproduction provided the original author(s) and source are given appropriate credit.

Received: January 4, 2017

Accepted: March 14, 2017

Citation: US Endocrinology, 2017;13(1):30-4

Corresponding Author: Fatima Younis Al Slail,

National Diabetes Prevention and Control Program, Ministry of Health, Prince Abdul Aziz Bin Mosaed Bin Jalawi Street, Riyadh, Saudi Arabia. E: fatima.alslail@gmail.com
Muslims represent $18-25 \%$ of the global population, which is approximately 1.1-1.5 billion people. There are about 50 million Muslims worldwide with diabetes who fast during the holy month of Ramadan each year. ${ }^{2}$ All healthy Muslims aged from late childhood or early adolescence or puberty (which ranges from 10-14 years and over) are commanded to fast from sunrise to sunset during the consecrated month of Ramadan. They must refrain from all oral intake of food, water, beverages, and medications during the fast and must also avoid sexual contact. ${ }^{3}$ Certain groups are exempted from fasting temporarily or permanently; the sick, the elderly, travelers, pregnant women, and breastfeeding women. ${ }^{4}$

Due to the nature of fasting, diabetic patients who fast have an increased risk of serious events, and these risks may rise in Ramadan due to the longer fasting periods. Fasting may lead to complications such as hypoglycemia, hyperglycemia with or without ketoacidosis, thrombosis, and dehydration. ${ }^{5}$ Thus, several guidelines have been formulated. ${ }^{6}$ However, there are gaps regarding diabetes management in Ramadan.

There is a detailed classification system for risk assessment of diabetic patients who fast during Ramadan, and the classification varies according to risk severity. There are several widely accepted compatibility standards for advice against fasting. Physicians should be aware of these situations when addressing and advising patients. However, asking the patient not to fast based on scientific evidence could not only lead to the patient fasting without telling their doctor but also may offend the cultural and religious values of the patient. ${ }^{3}$ Religious leaders, as well as healthcare professionals, should provide education and support for safer fasting during Ramadan. ${ }^{2}$

The American Diabetes Association recommends the use of patient-centered care (PCC) in the management of all diabetes cases. ${ }^{8}$ PCC is an approach that is sensitive towards the patient's preferences, needs, and values. ${ }^{9}$ Furthermore, Ramadan-focused education for diabetic patients has been proved to be essential in empowering them to change their lifestyles during Ramadan. ${ }^{10}$ 
Patients can face difficulties in maintaining their pre-Ramadan medication schedules, as they fear breaking their fast if they take them during the fasting period. This may result in some patients taking their medication too early, too late, or stopping them completely. Alternative routes of drug administration should be considered, as certain routes do not nullify fasting. Muslim scholars, medical practitioners, pharmacists, and specialists in other human sciences agree without opposition that topical routes, such as eye drops, ear drops, and sublingual tablets, such as nitroglycerin tablets (used for the treatment of angina), do not nullify fasting. In addition, subcutaneous (SC), intramuscular (IM), and intravenous (IV) medications do not affect fasting, unless it is intentionally administered for nourishment. ${ }^{11-13}$

Counseling before Ramadan is essential to all diabetic patients who are willing to fast during Ramadan, in order to adjust their medication timing, medication doses, and to optimize dietary changes and patterns of physical activity. Self-monitoring of blood glucose levels is especially important to detect acute symptoms. Most diabetic patients do not undertake preRamadan counseling, as some believe it is non-mandatory. Effort is required to convince diabetic patients who are aiming to fast to attend pre-Ramadan counseling visits. ${ }^{7,14-15}$

Thus, physicians should work for their patients to prepare a suitable and individualized diet and medication plan. ${ }^{3}$ All diabetic patients wishing to fast during Ramadan should receive detailed health advice 1-2 months before the start of Ramadan. The aim of this study is to assess the knowledge, attitude, and practices of physicians as well as identify issues related to diabetic patients wishing to fast during Ramadan.

\section{Methods}

Health services in Saudi Arabia are provided through different partners including public hospitals and primary healthcare centers (PHCCS), government health sectors, such as the Ministry of Health $(\mathrm{MOH})$, Military Health Services and University Health Institutions, which are structured to deliver free healthcare services to Saudi citizens. In addition to this, private sector health services, through its clinics and hospitals, constitute $31.1 \%$ of the total healthcare services in KSA in 2013.

A study protocol was submitted to the ethics committee of the Ministry of Health for approval; ensuring adherence to ethical principles such as those specified by the World Medical Association Declaration of Helsinki, and ethical approval was granted.

This study was qualitative in design and was conducted using two focus group discussions in primary healthcare (Etiga PHC). Each group included 12 participants who were physicians working in PHCCs. The two groups were categorized by gender; 12 males and 12 females were selected from 12 PHCCs by choosing one male and one female from each center. The physician age range was 30-57 years, and their clinical experience varied from 6-28 years.

The inclusion criteria were:

- Physicians working in PHCCs for more than five years.

- Physicians who independently manage more than 30 diabetic patients in a week.

- Two physicians should be selected from each PHCC.
The focus group discussion was conducted during Ramadan $1436 \mathrm{H}$ (June 2015). We developed a topic guide to discuss the health-seeking behavior of diabetic patients, that is, knowledge, attitude, physician practices, and capacity. The discussions were all conducted in English.

The participants who met the eligibility criteria and agreed to take part were enrolled. Each focus group session was attended by three investigators; one acted as the facilitator and two as note-takers. Participants were presented with a brief introduction describing the focus group process, the goals and objectives of the study, and explaining that sessions would be taped but participants would remain anonymous.

\section{Data collection}

The focus group discussions were conducted with physicians and healthcare providers attending PHCCs and diabetic centers. The discussion was conducted separately for males and females. All participants provided informed consent. There was no risk involved in this study because no intervention or invasive tests were performed. The study participants were not given any monetary or compensatory benefits. There were a tape recorder and two assistants who transcribed the conversation during the discussion.

\section{Analysis}

We transcribed the tape recorder and compared the results with the notes taken by the assistants to complete any missing words. We used the raw data to categorize, coding the responses from the participants according to their contexts and relevance for the research question, and performed a thematic analysis. Patterns emerged during the systematic coding process, and themes were then determined by the researchers according to concepts and issues that the participants emphasized repeatedly within groups and between groups. All authors were involved in the analysis.

\section{Results}

\section{Knowledge}

A subgroup of physicians was aware of the therapeutic regimens for fasting that are supported by the American Diabetes Association, which include a healthy diet, exercise, and a lower glycemic index. The majority of the respondents agreed that glitazones should be prescribed as two-thirds of a dose in the morning and then one-third in the evening. The respondents also stated that short-acting secretagogues and sulphonylurea are not recommended. However, two respondents stated that the dose of sulphonylurea should be prescribed at half the dose in Suhoor (eating late at night in preparation for fasting) and half the dose in Eftar.

\section{Whether to nullify fasting}

All respondents agreed that prescribed mouthwashes, gargles, and oral sprays would not break the fast, unless they are swallowed. Furthermore, ear drops will not nullify fasting unless the eardrum is perforated. Physicians claimed that all substances absorbed into the skin, such as creams, ointments, and patches, do not affect fasting. However, a nutrient substance could nullify the fast. Moreover, respondents stated that vaginal pessaries, tablets, ovules, and douches did not break the fast.

The respondents stated that $\mathrm{SC}, \mathrm{IM}$, and IV medications do not affect the fast unless it is provided for nourishment. The physicians also agreed that anesthesia can nullify the fast (the gas itself does not nullify fasting) because the patient will be unconscious, and Hejama (i.e., cupping-blood 
Table 1: Examples of risk assessment questions

Categorize the diabetic patients according to risk, and what you would advise
regarding fasting in the following medical conditions?
Risk level: 1 = very high risk; 2 = high risk; $3=$ moderate risk; $4=$ low risk
1. Severe hypoglycemia within the last three months prior to Ramadan
2. Patient with a history of recurrent hypoglycemia
3. Patient with hypoglycemia unawareness
4. Patient with sustained poor glycemic control
5. Ketoacidosis within the last three months prior to Ramadan
6. Type 1 diabetes, acute illness
7. Hyperosmolar hyperglycemic coma within the previous three months
8. Patient who performs intense physical labor or is pregnant
9. Patient on chronic dialysis
10. Patient with moderate hyperglycemia (average blood glucose between 150
and 300 mg/dl, A1c $7.5-9.0 \%$ )
11. Patient with renal insufficiency
12. Patient with advanced macrovascular complications
13. Patient living alone who is treated with insulin or sulfonylureas
14. Patient living alone, patient with comorbid condition that presents additional
risk factors
15. Patient in old age with ill health, drugs that may affect mentation (cognitive
function)
16. Well-controlled patient treated with short-acting insulin secretagogues such
thiazolidinedione, who is otherwise healthy

drawn by vacuum from a small skin incision for therapeutic purposes) nullifies fasting, as stated in the Sunnah (all the traditions and practices of the Islamic prophet that have become models to be followed by Muslims). Furthermore, sublingual nitroglycerine tablets placed under the tongue for the treatment of angina does break the fast because patients swallow the tablet with saliva.

There is controversy regarding whether the use of nasal drops, spray, inhalers, suppositories, or enemas can nullify fasting. There was a small group of respondents who stated that these treatments affect the fast.

\section{Risk assessment for fasting diabetic patients}

The respondent assessments for the categorization of diabetic patient risks (see Table 1) found that the majority lacked knowledge with respect to the following patient situations: patients who perform intense labor, pregnancy, type 1 diabetes, patients with sustained poor glycemic control, acute illness, patients with hypoglycemia unawareness, patients with history of recurrent hypoglycemia, and patients on chronic dialysis.

All high-risk patients should be advised not to fast the entire month of Ramadan. The majority of doctors advised their patients to abstain from fasting, except for patients with type 1 diabetes and patients who perform intense physical labor.

All physicians reported that they advised patients to abstain from fasting because they were more aware than the Mufti (an Islamic scholar who infers and explains Islamic law) about the risks pertaining to their medical conditions (see Table 2). A group of respondents stated that they agreed, but the advice is mainly for type 1 diabetes patients and those who experience hypoglycemia in the afternoon.
Table 2: Examples of attitude and practices questions

\author{
Have you asked patients not to fast? \\ Do patients consult you before Ramadan? \\ Do you talk to your patients regarding management of diabetes during Ramadan? \\ What changes do you suggest to patients before Ramadan? \\ Does the physician go to the healthcare facility on regular basis during Ramadan? \\ Are laboratory, pharmacy and other facilities regularly available? \\ According to your experience, does the health of diabetic patients improve or \\ deteriorate after Ramadan?
}

Traditionally, Muslims break their fast with dates. Several respondents indicated that they advised their patients to break their fasting with no more than three dates. However, other physicians did not recommend breaking the fast with dates. Furthermore, they stated patients perceived this as a punishment because it is a holy tradition.

The majority of doctors advised patients to avoid eating honey. However, if patients insisted, they were allowed to have 3-4 $\mathrm{ml}$ because it has a high sugar concentration. The physicians provided patients with consultations before Ramadan but limited their consultations to patients with appointments.

All respondents believed it was important to provide advice at each visit, such as testing blood sugar four times a day and trying to fast three days before Ramadan prior to the decision to fast for the entire month. Patients using insulin have critical periods between Duhur (midday) and Asr (afternoon). Furthermore, they had issues with uncontrolled hyperglycemia and reduced physical activity.

The respondents suggested that, in preparation for Ramadan, they preferred their patients use analogue insulin 50/50, but this option is limited in availability in Saudi Arabia. One approach is to divide the dose between Suhoor and Eftar. The physicians permitted use of metformin any time because it does not cause hypoglycemia. Short-acting insulin should be taken immediately after Eftar. They all agreed that physical activity should occur after Eftar and near Taraweeh (night prayer).

\section{Issues faced by the physician}

The issues faced by physicians before and during Ramadan include how to adjust the dose and convince the patient to follow a new regimen. Additionally, patients may refuse to change their diabetic treatment from tablets to insulin. The follow-up of gestational diabetic patients in PHCCs requires one month before they are referred to a specialized diabetic clinic.

Furthermore, there is a loss of patient follow-up due to referral to the hospital, shifting of doctors, poor services, fear of hypoglycemia, and a lack of training courses about diabetes for patients before and during Ramadan. Similarly, the lack of a dietitian at PHCCs and the lack of feedback from hospitals are obstacles that cause loss of follow-up.

The following issues were reported in elderly patients: a refusal of the laboratory to perform an examination for patients referred from another PHCC, high patient flow with limited resources and time for patients, and long waiting times for examinations. Medications were only available for eligible patients. An additional issue during Ramadan was the lack of 
medication compliance due to fear of hypoglycemia. Furthermore, there was inconsistency in doctors following the same patient during visits. All respondents agree that the flow of patients visiting PHCCS is reduced in Ramadan compared with other months. The explanations for this phenomenon include the following:

- Patients have appointments during Shaban and take their prescriptions for two months, but they do not show up during Ramadan.

- Many patients travel to their hometown during the Ramadan holiday.

- In case of an emergency, patients go to hospitals during Ramadan instead of visiting PHCCs, although there is an emergency unit in each PHCC clinic.

- Working hours change during Ramadan.

- Female patients cook before Eftar (breaking the fast), and after Eftar, therefore there is no one to bring them to appointments, so they are not able to attend.

- Misconceptions about blood testing exist, as patients believe that blood tests break the fast.

The respondents indicated that doctors are available in their clinics. However, pharmacists are not available at all times during Ramadan. Additionally, the shortage of nursing staff is a problem all year.

There was controversy regarding the improvement of the health of diabetic patients during Ramadan. A group of respondents stated that the health of most patients deteriorated (e.g., increased weight, less activity, eating a variety of unsuitable foods). Conversely, a group of physicians stated that patient health remained the same before and after Ramadan (i.e., patients in good control before Ramadan had good control during Ramadan).

A physician claimed that medical training on diabetes management conducted during Ramadan or any workshop conducted before Ramadan "mainly in Shabaan" is sponsored by either the Riyadh authority or drug companies (see Table 3). There is no training during Ramadan, and they do not receive any medical information on the management of diabetes during Ramadan. All the respondents suggested they need training in the management of diabetes during Ramadan.

The physicians indicated that diabetic patients can prepare for diabetes management during Ramadan through the following channels:

- Health education in the form of workshops or face to face during visits (regarding diet, dose and physical activity).

- Healthy diet commercials.

- Specialized teams in each health center (physician/nutritionist/diabetic nurse and health educator) to choose the optimum dose for patients.

- Patient follow-up is lost between the health center and the general hospital when the patient referred to the hospital has a long wait time for an appointment (approximately two months).

- There should be an electronic record for the patient because the patient may forget the name of the treatment he or she receives. Additionally, linking this record between the PHCC and the hospital will prevent the loss of patient follow-up and feedback processes will be created.

\section{Discussion}

Several guidelines have been formulated. ${ }^{5}$ However, there are gaps regarding diabetes management during Ramadan. These issues
Table 3: Examples of questions regarding capacity

Have you received any medical training on management of diabetes during fasting?

Have you received any medical information on management of diabetes during fasting in Ramadan?

What can be done for diabetics to prepare them for management of diabetes during Ramadan?

can be addressed by improving physician awareness of the probable risks associated with fasting during Ramadan and how to eliminate those risks.

Patients known to be at high or moderate risk are missed by the practicing doctor because they did not identify the following patient groups: patients with moderate hyperglycemia, patients with renal insufficiency, patients with advanced macrovascular complications, patients living alone who are treated with insulin or sulfonylureas, patients living alone, patients with comorbid conditions that present additional risk factors, patients with comorbid conditions that present additional risk factors, and the elderly with ill health. As a result, these patients may not receive the correct advice regarding fasting.

Implementing a detailed classification system for risk assessment by a physician of diabetic patients who fast during Ramadan, influences knowledge of the practical management of diabetes and identifying patients who are allowed to fast. ${ }^{6.17}$ The physicians should also be aware of the drugs that nullify fasting. All the respondents had misconception regarding nitroglycerin tablets placed under the tongue nullifying fasting which is contrary to other evidence in the literature. ${ }^{1-13}$

Managing diabetic patients is a challenge, and it is a sensitive issue to advise patients whether or not to fast. Furthermore, it may offend their cultural and spiritual values. ${ }^{3}$ Thus, it is also essential to address religious awareness in a delicate way.

Furthermore, it would be valuable to patients undergo pre-Ramadan assessment with education using a structured program that covers all the aspects of diabetes related to physical activity, meal planning, glucose monitoring, dosage, and timing of medication. Education will also improve the understanding of fasting with diabetes and will help individuals to assess their risks and options to achieve fasting safety.

Physicians indicated that they face difficulty convincing patients to visit them before Ramadan as patients appear only for taking medication. Thus, changing patient behavior toward the clinic visit by empowering the patients through implementing the recommendations of the American Diabetes Association regarding the use of PCC and Ramadanfocused pre-Ramadan counseling would have a great value, on the long run. ${ }^{8,10}$

Diabetic patients break their fast with dates, as reported by physicians. This is the norm during Ramadan, with the recommendation not to exceed three dates. Moreover, doctors advise the same patients to avoid eating honey. The majority of health problems probably arise from following an improper diet or as a result of overeating and insufficient sleep. ${ }^{2}$ Both 
physicians and nutritionists have essential roles in educating patients. Educating patients about diet and the calories contained in foods, as well as selecting and consuming an energetically balanced diet positively influences food choices. ${ }^{18,19}$

The medical staff at PHCCs face additional pressures during Ramadan due to shifting work hours, a lack of male nurses, and the limited number of pharmacists. Thus, it can lead to significant burdens for healthcare providers and healthcare facility finances and personnel. ${ }^{10}$ The role of PHCCs could be improved by adding diabetic educators and nutritionists. This will eventually lead to improvement in quality and potentially the quantity of care delivered to patients treated at PHCCS. Moreover, this can result in improved patient gratification and patient instruction about the management of diabetes during Ramadan.

PHCCs have impediments regarding the limited availability of laboratory testing all over the week before, during, and after Ramadan. This is can be solved by identifying the reasons why laboratory testing is only available once a week. The abundance of laboratory testing is crucial for running the visits smoothly. PHCCs will need to raise this issue with the $\mathrm{MOH}$ for convenient health services.

We also found that several diabetic medications were not always available.This indicates the necessity of creating an early warning system for impending medical supplies and drug product shortages that provides ample opportunity to prepare for all implications of the shortage. ${ }^{10}$ Furthermore, identifying and obtaining medications or alternative medical products should be done rapidly by the health system to prevent disturbances in healthcare and to provide continuous, therapeutically equivalent, safe drug treatment at comparable costs. ${ }^{10}$

Some respondents recommended that medical records from PHCCs and hospitals should be linked through an electronic health system. This will improve the referral system and keep patients' information records constantly accessible and modifiable between the different care levels.

\section{Strengths and limitations}

The strength of our study was the diverse range of respondents in terms of physician age, experiences, and selected PHCCs locations. All respondents described the current situation with honesty, which reflects the magnitude of the problem.
There are limitations in this study because it was not possible to cover all aspects of this particular issue. This study did not explore in depth the views of physicians working outside Riyadh. Future research should investigate the views of patients to identify further barriers to research participation.

\section{Recommendations}

Physicians in PHCCs need scheduled training activities through workshops, conferences, and meetings, with a continuous evaluation of their judgment in classifying the diabetic patient as low, moderate, high, and very high risk. Physicians must decide if diabetic patients should fast. The workshop should also cover the tests and treatment methods that can nullify fasting.

Physicians also need further training on patient counseling, which has a positive influence on changing the perspectives of patients and can improve regimen adherence and control low blood sugar levels. Patients should also receive information regarding calorie counting and diet in the patient management plan during follow-up visits.

The health authority should assess PHCCs strategies to prevent a lack of medical supplies. For example, laboratories and pharmacies need to create an early warning system with alerts in advance of impending lab and drug product shortages. This system will measure the level of preparedness and readiness of PHCCS to meet health service needs.

Health centers should be empowered with efficient human resources to ensure a fully functioning facility. There are occasional shortages of chronic disease doctors, laboratory technicians, pharmacists, and female nurses. Male nurses, nutritionists, and health educators need to be involved in the hierarchy for improving healthcare services.

The high turnover of PHCC doctors can be reduced by long-term contracts, including benefits to ensure doctor sustainability and hence better doctor-patient relationships. It is essential to establish and implement referrals between all levels within the healthcare network to improve patient follow-up.

The recommendations provided by the groups will be forwarded to higher authorities. This study will improve our understanding of beliefs and determine the awareness level of diabetes management in a physician population. The health authority will refine their strategic plan for enhancing the quality of health care for diabetes management during Ramadan.
1. An Analysis of the World Muslim Population by Country/Region, 2005. Available at: www.factbook.net/muslim_pop.php (accessed 2005. Available 14,2005 .

2. Bravis V, Hui E, Salih S, et al., Ramadan Education and Awareness in Diabetes (READ) programme for Muslims with Type 2 diabetes who fast during Ramadan, Diabet Med, 2010;27:327-31.

3. Niazi AK, Kalra S, Patient centred care in diabetology: An Islamic perspective from South Asia, J Diabetes Metab Disord, 2012:11:30. The Holy Quran, Sura 2: Verses 183-185.

5. Salti I, Benard E, Detournay B, et al., A population-based study of diabetes and its characteristics during the fasting month of Ramadan in 13 countries: Results of the epidemiology of diabetes and Ramadan (EPIDIAR) study, Diabetes Care, 2004;27:2306-11.

6. Al-Arouj M, Bouguerra R, Buse J, et al., Recommendations for management of diabetes during Ramadan, Diabetes Care, 2005;28:2305-11.

7. Beshyah S, Benbarka M, Sherif I, Practical management of diabetes during Ramadan fast, Libyan J Med, 2007:2:185-9.
8. Inzucchi SE, Bergenstal RM, Buse JB, et al., Management of hyperglycemia in type 2 diabetes: a patient centered approach, Diabetes Care, 2012:35:1364-79.

9. Committee on Quality of Health Care in America: Institute of Medicine, Crossing the Quality chasm: A New Health System for the 21st Century, Washington, DC: The National Academies Press; 2001

10. Fatim J, Karoli R, Chandra A, Naqvi N, Attitudinal determinants of fasting in type 2 diabetes mellitus patients during Ramadan, I Assoc Physicians India, 2011:59:630-4.

11. Ramadan Health and Spirituality Guide, 2006. Available at: http:// ebookee.org/Ramadan-Health-and-Spirituality-Guide_132743.html (accessed April 2, 2013).

12. Ali R, Siddiqui $H$, Anjum Q, et al., Knowledge and perception of patients regarding medicine intake during Ramadan, JCPSP, 2007;17:112-3.

13. Aadil N, Houti IE, Moussamih S, Drug intake during Ramadan, BMJ, 2004;329:778-82.
14. Qureshi B, Diabetes in Ramadan, $J$ R Soc Med, 2002;95:489-90.

15. Fox ER, Birt $A$, James $K B$, et al., ASHP guidelines on managing drug product shortages in hospitals and health systems, Am J Health Sys Pharm, 2009;66:1399-406.

16. Colliers International, Kingdom of Saudi Arabia Healthcare Overview, 2012. Available at: www.colliers.com// media/961B1B350CF146FE910AFDBECDCC605A.ashx (accessed May 25, 2015).

17. Al-Arouj M, Assaad-Khalil S, Buse J et al., Recommendations for management of diabetes during Ramadan, Diabetes Care, 2010;33:1895-902

18. Zarkin GA, Dean N, Mauskopf JA, Williams R, Potential health benefits of nutrition label changes, Am J Public Health, 1993;83:717-24.

19. Center for Science in the Public Interest: Menu labeling Available at: www.cspinet.org/menulabeling/ (accessed July 22, 2009). 